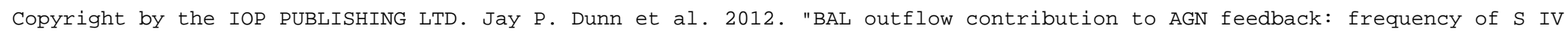
outflows in the SDSS," ApJ 750143 doi:10.1088/0004-637X/750/2/143

\title{
BAL OUTFLOW CONTRIBUTION TO AGN FEEDBACK: FREQUENCY OF S IV OUTFLOWS IN THE SDSS
}

\author{
Jay P. Dunn ${ }^{1}$, Nahum Arav ${ }^{2}$, Kentaro Aoki $^{3}$, Ashlee Wilkins ${ }^{4}$, Courtney Laughlin ${ }^{2}$, \\ DOUG EDMONDS $^{2}$, AND MANUEl BAUTISTA ${ }^{5}$ \\ ${ }^{1}$ Department of Chemistry and Physics, Augusta State University, Augusta, GA 30904, USA; jdunn10@aug.edu \\ ${ }^{2}$ Department of Physics, Virginia Tech, Blacksburg, VA 24061, USA; arav@vt.edu, edmonds@vt.edu \\ ${ }^{3}$ Subaru Telescope, National Astronomical Observatory of Japan, 650 North A'ohoku Place, Hilo, HI 96720, USA; kentaro.aoki@ hawaiiantel.net \\ ${ }^{4}$ Cornell University, Ithaca, NY 14853, USA; anw37@ cornell.edu \\ ${ }^{5}$ Department of Physics, Western Michigan University, Kalamazoo, MI 49008-5252, USA; manuel.bautista@ wmich.edu \\ Received 2010 September 23; accepted 2012 February 16; published 2012 April 25
}

\begin{abstract}
We present a study of broad absorption line (BAL) quasar outflows that show S IV $\lambda 1063$ and S IV* $\lambda 1073$ troughs. The fractional abundances of S IV and C IV peak at similar value of the ionization parameter, implying that they arise from the same physical component of the outflow. Detection of the S IV* troughs will allow us to determine the distance to this gas with higher resolution and higher signal-to-noise spectra, therefore providing the distance and energetics of the ubiquitous C IV BAL outflows. In our bright sample of 156 SDSS quasars, $14 \%$ show C IV and $1.9 \% \mathrm{~S}$ IV troughs, which are consistent with a fainter magnitude sample with twice as many objects. One object in the fainter sample shows evidence of a broad S IV trough without any significant trough present from the excited state line, which implies that this outflow could be at a distance of several kpc. Given the fractions of $\mathrm{C}$ IV and S IV, we establish firm limits on the global covering factor on S IV that ranges from $2.8 \%$ to $21 \%$ (allowing for the $k$-correction). Comparison of the expected optical depth for these ions with their detected percentage suggests that these species arise from common outflows with a covering factor closer to the latter.
\end{abstract}

Key word: quasars: absorption lines

Online-only material: color figures

\section{INTRODUCTION}

Quasar outflows are detected as blueshifted absorption troughs with respect to the active galactic nucleus (AGN) restframe spectrum. These outflows carry mass, momentum, and energy into the surrounding environment, and therefore may be important in the context of AGN feedback (Arav et al. 2012, in preparation). To quantify the effect of the outflows on their environment we need to determine their average mass flow rate $(\dot{M})$ and associated kinetic luminosity $\left(\dot{E}_{k}\right)$. Assuming the outflow is in the form of a partial thin, spherical shell (see Arav et al. 2012, in preparation, Section 5.2 and their Figure 4) moving with a constant radial velocity $v$ at a distance $R$ from the central source, these quantities are given by

$$
\dot{M} \sim 4 \pi \mu m_{p} \Omega R N_{\mathrm{H}} v, \quad \dot{E}_{k}=\frac{1}{2} \dot{M} v^{2},
$$

where $N_{\mathrm{H}}$ is the total column density of hydrogen, $m_{p}$ is the mass of a proton, $\mu=1.4$ is the plasma's mean molecular weight per proton, and $\Omega$ is the fraction of the solid angle around the quasar occupied by the outflow.

Over the past few years, we have built a research program to determine $\dot{M}$ and $\dot{E}_{k}$ in quasar outflows (Arav et al. 2008; Korista et al. 2008; Moe et al. 2009; Dunn et al. 2010; Bautista et al. 2010). Targeting objects that show absorption troughs from excited and/or metastable levels, we were able to obtain measurements of $N_{\mathrm{H}}$ and $R$ to an accuracy of $\sim 30 \%$. The outflow's velocity is determined simply and accurately from its blueshift in the spectrum, which leaves $\Omega$ as the major uncertain quantity in Equation (1). No information regarding $\Omega$ can be obtained from a single object's spectrum, as we only see the absorption along the line of sight (LOS). Therefore, our inference regarding $\Omega$ is statistical in nature. Essentially, we use the detection rate of objects with outflows among all quasars as an estimate for $\Omega$. (See discussion in Dunn et al. 2010, Arav et al. 2012, in preparation, and Section 5 here.)

A significant uncertainty in our case stems from the fact that we can only determine distances to outflows that show troughs from excited and metastable levels. For most of our objects, the spectra cover such transitions only from singly ionized species (e.g., Fe II and Si II). However, the majority of outflows only show troughs from high-ionization species (HiBALs) where in both optical (Hewett \& Foltz 2003) and IR (Dai et al. 2008) surveys, $20 \%$ of all quasars are inferred to have C Iv broad absorption lines (BALs). In optical surveys, low-ionization BALs (LoBALs) are quite rare, only $0.5 \%$ of the Sloan Digital Sky Survey (SDSS) quasars show LoBALs (Trump et al. 2006). However, recent work (Dai et al. 2010) have shown that in IR surveys (that are much less sensitive to obscuration) $4 \%$ of all quasars are LoBALs. While these findings considerably narrow the gap between the two populations, we still need to address the question regarding the distance and energetics of both outflow manifestations. Are the LoBALs results representative for the HiBALs, which are majority of the outflows?

There are good indirect arguments that outflows which exhibit LoBALs are regular $\mathrm{C}$ IV outflows viewed through a high opacity LOS that allows for the formation of singly ionized troughs. One plausible example would be when the outflows see an ionizing continuum that is filtered by the edge of the putative AGN torus (Hall et al. 2003; Dunn et al. 2010; Arav et al. 2012, in preparation). In that case, their $\Omega$ and $R$ will be the same as that of the C IV BALs. Alternatively, the lower detection rate can be explained as LoBALs being a different kind of an outflow, with $\Omega \simeq 0.04$ on average and possibly no correspondence with the $R$ of HiBAL outflows. 
A direct way to advance on this issue is to observe excited state troughs from higher ionization species. Thus, a simple comparison with the ubiquitous C IV is feasible and we circumvent the ambiguity due to the preferred ionization state of the outflow. Such troughs appear at wavelengths shortward of Ly $\alpha$ but have been rarely analyzed in the literature due to blending with the thick Lyman forest absorption at high redshifts.

Here, we target the SIV/S IV* $\lambda \lambda 1062.66,1072.97$ lines (the $\mathrm{S} \mathrm{IV}^{*} \lambda 1072.97$ arises from the metastable $3 s^{2} 3 p^{2} P_{3 / 2}^{o}$ level). Absorption troughs due to S IV were seen previously in BALQSOs PG 0946+301 (Arav et al. 1999, 2001) and WPVS007 (Leighly et al. 2009), as well as in the Seyfert 1 galaxy NGC 4151 (Kraemer et al. 2006). The fractional abundance of S IV and C IV peak at similar value of the ionization parameter, implying that they arise from the same physical component of the outflow (see Section 3 and Figure 3). Therefore, measuring $N_{\mathrm{H}}$ and $R$ for these outflows will yield less modeldependent measurements for $\dot{M}$ and $\dot{E}_{k}$ in the majority of outflows. Here, we take the first step in determining the $M$ and $\dot{E}_{k}$ for these outflows by establishing limits on $\Omega$.

The plan of the paper is as follows. In Section 2, we describe our SDSS sample selection, outline the detection methods, and present the detected S IV outflows. In Section 3, we elaborate on the relationship between the detection rate of S IV vs. that of C IV, Si IV, and C II troughs in quasar outflows. In Section 4, we discuss our results and their implications with regards to $\Omega$. Finally, we summarize our findings in Section 5.

\section{SURVEY}

\subsection{Criteria for Detecting S IV Absorption Outflows in High-redshift Quasars}

Our sample takes advantage of the wealth of archived data from the SDSS conducted with a $2.5 \mathrm{~m}$ telescope at Apache Point Observatory (York et al. 2000). We search for quasars through the SDSS data release 7 (Schneider et al. 2010) and download the calibrated spectra via the online SQL interface to the Catalog Archive Server. We begin by introducing two-key selection criteria. Our first criterion is to include only quasars brighter than an $r^{\prime}$ magnitude of 18. We constrain the $r^{\prime}$ magnitude in order to keep the signal to noise $(\mathrm{S} / \mathrm{N})$ in the Ly $\alpha$ forest region high enough to minimize confusion with spurious troughs. To observe S IV $\lambda 1063$, given the $3800 \AA$ short wavelength limit of the SDSS, the second criterion is that the redshift of the objects must be $z>2.8$. These two limits result in a total of 156 quasars (after excluding objects misidentified as quasars based on spectral properties).

We search the data first for C IV absorption because: (1) we do not expect to find absorption from $\mathrm{S}$ IV unless the ubiquitous $\mathrm{C}$ IV trough is present, (2) the CIV spectral region is free of Ly $\alpha$ forest contamination, and (3) it is the natural benchmark for assessing the overall percentage of outflows in quasar. Our search criteria are that the full width at half-maximum (FWHM) of the absorption must be greater than $500 \mathrm{~km} \mathrm{~s}^{-1}$ and there must be an outflow velocity between 0 and $-7000 \mathrm{~km} \mathrm{~s}^{-1}$. The first criterion allows us to select against associated and intervening $\mathrm{C}$ IV systems, and at the same time, yields a wide enough S IV trough that can be differentiated from Ly $\alpha$ forest troughs (see below). The second criterion prevents confusion due to overlap of the $\mathrm{S}_{\mathrm{IV}}$ troughs with absorption troughs arising from the $\mathrm{O}$ VI doublet $(\lambda \lambda 1032,1038)$.

At a redshift of $z \sim 3$, we expect the number of Ly $\alpha$ absorption systems in any given sight line to a quasar, within our wavelength range of (1000-1216) $\AA$, to be $\sim 150$ (Bechtold 1994). However, we can distinguish intrinsic S IV absorption systems of the quasar from the Ly $\alpha$ forest absorbers with the following criteria: (1) the coincidence in velocity with C IV, and even more so with absorption troughs from Si IV (see Section 2.2 and Figure 1), is our primary method of discerning the origin of the absorbing gas, and (2) the velocity width of the absorption trough. This criterion relies on the actual trough widths. Typically, Ly $\alpha$ forest absorbers have an FWHM $\lesssim 25 \mathrm{~km} \mathrm{~s}^{-1}$ (Outram et al. 1999; Danforth \& Shull 2008). The spectral resolution of SDSS is $\sim 150 \mathrm{~km} \mathrm{~s}^{-1}$, and therefore the Ly $\alpha$ forest absorbers are not resolved. However, an FWHM of $150 \mathrm{~km} \mathrm{~s}^{-1}$ is more than a factor of three smaller than our FWHM cutoff. Thus, only chance alignments of several Ly $\alpha$ absorbers with the C IV absorption in velocity space can be misidentified. (3) Our final criterion is the presence of a trough from the excited state transition $\mathrm{S} \mathrm{IV}^{*}$ $\lambda 1073$, covering a similar velocity range. Kinematic agreement between troughs from S IV* $\lambda 1073$ and S IV $\lambda 1063$ with troughs not contaminated by Ly $\alpha$ forest lines (e.g., C IV and Si IV) makes false detections due to $\operatorname{Ly} \alpha$ lines significantly less likely.

\subsection{Quasars with SIV Absorption Troughs}

Given the above selection criteria, we find that of the 156 quasars, 25 have C IV troughs with an FWHM $>500 \mathrm{~km}^{-1}$ or $\sim 16 \%$ of the total (see Table 1 ). We note though that three of these show very weak N v troughs and strong Si II troughs and are likely associated or intervening absorption systems and not outflows from the quasar. We omit these three objects from further discussion, which yields a more conservative value for the fraction of C IV outflows of $14 \%$. We also examined the spectra for outflow troughs from Si IV and C II. In the survey, 17 of the objects that show C IV outflow troughs have corresponding Si IV outflow troughs or $11 \%$ from the full sample. There are 13 objects that show $\mathrm{C}$ II absorption troughs. We discuss the detection percentage of all the ions in Section 3.

Of the 156 objects with the proper search criteria, we find three robust detections of S IV and $\mathrm{S} \mathrm{IV}^{*}$ absorption that appear to be kinematically associated with the C IV and Si IV troughs. One other object, SDSS J1319+4456, appears to have a trough from S IV with an FWHM of approximately $510 \mathrm{~km} \mathrm{~s}^{-1}$, with no associated $\mathrm{SIV}^{*}$ trough. These less robust features warranted further scrutiny, which led us to obtain a higher resolution spectrum using the Subaru telescope. We find that this case is a superposition of three Ly $\alpha$ forest troughs and we omit it from the S IV sample. The other three objects are supported by significantly broader S IV troughs (too broad for the superposition of two or three $\operatorname{Ly} \alpha$ troughs) kinematically matching SIV* troughs, which makes the possibility of false detections highly unlikely.

For trough measurement techniques and values for the outflows in the remaining three objects, see the Appendix. We present the far-UV and near-UV regions of the SDSS spectra for the three S IV detections in Figure 1. To illustrate the existence of S IV and S IV* troughs, we include in Figure 1 a scaled apparent optical depth template $\left(I=e^{-\tau_{\text {app }}}\right.$, where $I$ is the normalized intensity) of the Si IV $\lambda 1403$ trough. We do so for both the $\mathrm{S}$ IV $\lambda 1063$ and $\mathrm{S} \mathrm{IV}^{*} \lambda 1073$ lines, where we allow no freedom in the wavelength position as it corresponds exactly to the velocity structure of the Si IV trough. We use a single scaling factor for each Si IV $\tau_{\text {app }}$ template to match the data. These three objects give a conservative fraction of $1.9 \%$ for S IV outflows in this sample. 

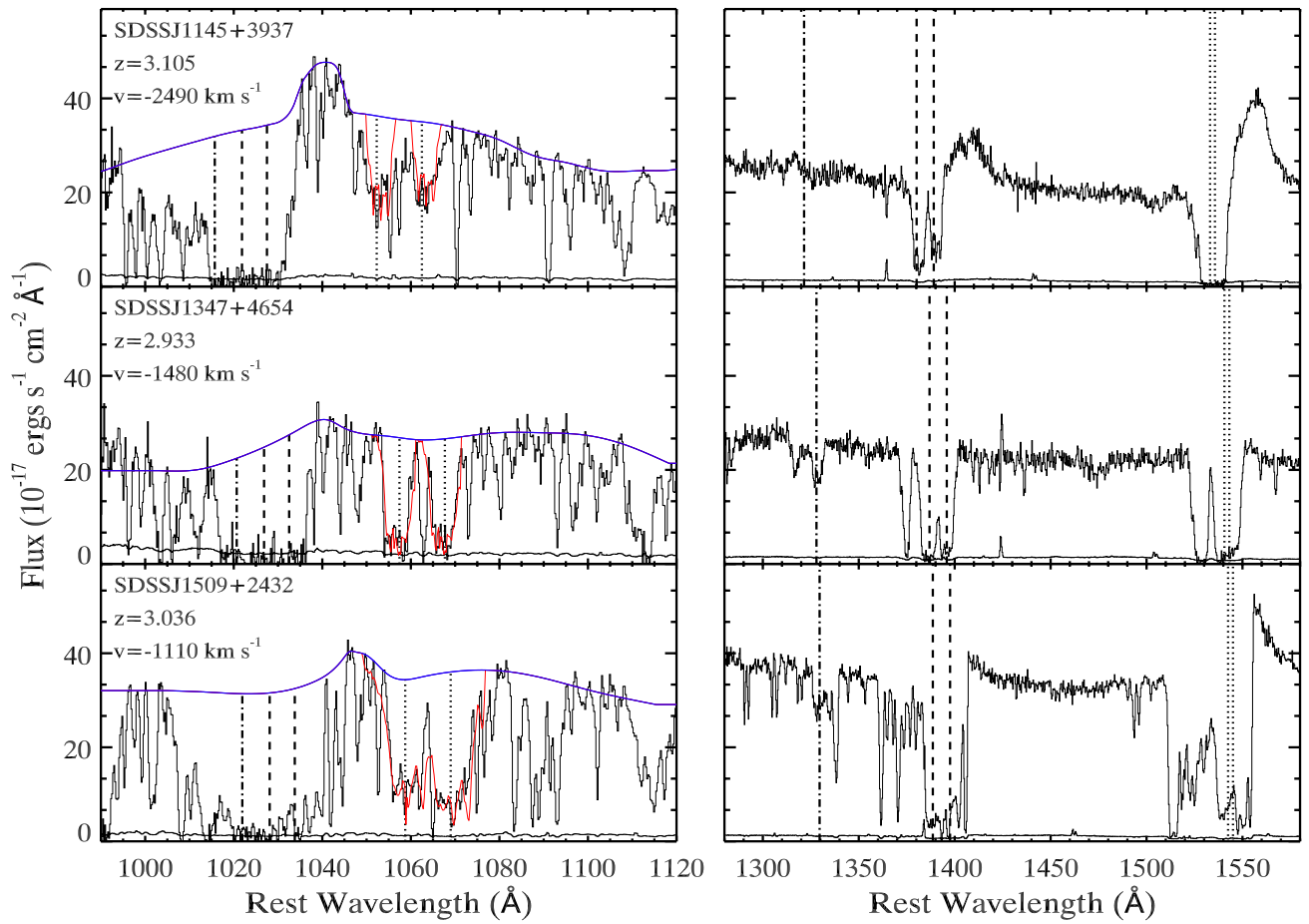

Figure 1. Left: far-UV spectra for the three objects that show $\mathrm{S}$ IV and $\mathrm{S} \mathrm{IV}^{*}$ absorption. The object name, redshift, and central trough velocity are listed at the upper left of each panel. The dotted vertical lines mark the location of the S IV and S IV* absorption troughs, while dashed lines mark the position of the respective O vI doublet $\lambda \lambda 1032,1038$ positions and the dashed-dotted lines the position of Ly $\beta \lambda 1026$. The solid blue curve shows a spline fit approximation of the continuum in the region. In red, we show a scaled optical depth template of the Si IV $\lambda 1403$ trough, placed at the expected position of the S IV $\lambda 1063$ and S IV* $\lambda 1073$ lines (see the text). Right: near-UV spectra for the same objects. The C IV troughs from $\lambda \lambda 1548,1551$ are marked with dotted lines, the Si IV doublet $\lambda \lambda 1394,1403$ with a dashed lines, and the expected position of the $\mathrm{C}$ II $\lambda 1335$ line, with a dashed-dotted line.

(A color version of this figure is available in the online journal.)

Table 1

Quasars with C IV Outflows in Our Sample

\begin{tabular}{|c|c|c|c|c|c|c|}
\hline SDSS Name & Plate-MJD-Fiber $^{\mathrm{a}}$ & $r^{\prime}$ Magnitude & $z^{\mathrm{b}}$ & $\mathrm{SIV}^{\mathrm{c}}$ & $\mathrm{Si}_{\mathrm{IV}}^{\mathrm{c}}$ & $\mathrm{C} \mathrm{II}^{\mathrm{C}}$ \\
\hline J083535.69+212240.22 & 53349-1929-041 & 17.89 & 3.116 & & & \\
\hline J084401.95+050357.92 & $52650-1188-464$ & 17.88 & 3.346 & & $\mathrm{y}$ & $\mathrm{y}$ \\
\hline $\mathrm{J} 100841.22+362319.36$ & 52993-1426-041 & 17.68 & 3.125 & & & \\
\hline $\mathrm{J} 102009.99+104002.79$ & 52999-1597-040 & 17.77 & 3.167 & & & \\
\hline $\mathrm{J} 102025.28+334633.42$ & 53442-1955-522 & 17.96 & 2.940 & & $\mathrm{y}$ & $\mathrm{y}$ \\
\hline J103419.71+191222.28 & 53770-2376-139 & 17.26 & 3.160 & & $\mathrm{y}$ & \\
\hline J105101.21+153226.88 & 53852-2483-257 & 17.93 & 2.820 & & $\mathrm{y}$ & \\
\hline J112258.77+164540.31 & 54176-2499-308 & 17.78 & 3.031 & & $\mathrm{y}$ & $\mathrm{y}$ \\
\hline $\mathrm{J} 113008.19+535419.90$ & 52707-1014-211 & 17.82 & 3.059 & & $\mathrm{y}$ & $\mathrm{y}$ \\
\hline J114323.71+193448.06 & $54180-2509-273$ & 17.74 & 3.347 & & $\mathrm{y}$ & $\mathrm{y}$ \\
\hline J114548.38+393746.70 & $53442-1997-450$ & 17.93 & 3.105 & $\mathrm{y}$ & $\mathrm{y}$ & \\
\hline $\mathrm{J} 115023.58+281907.50$ & $53793-2223-376$ & 16.96 & 3.132 & & $\mathrm{y}$ & \\
\hline $\mathrm{J} 120934.53+553745.70$ & 52707-1019-350 & 17.83 & 3.559 & & & \\
\hline J131048.17+361557.75 & 53799-2016-541 & 17.88 & 3.395 & & & \\
\hline J131927.57+445656.54 & 53089-1376-380 & 17.55 & 2.978 & & $\mathrm{y}$ & \\
\hline $\mathrm{J} 131912.40+534720.58$ & 52724-1041-271 & 17.85 & 3.091 & & $\mathrm{y}$ & \\
\hline J134722.83+465428.58 & 52736-1284-140 & 17.97 & 2.933 & $\mathrm{y}$ & $\mathrm{y}$ & $\mathrm{y}$ \\
\hline J141321.05+092204.91 & 53794-1810-516 & 17.75 & 3.345 & & & \\
\hline $\mathrm{J} 145125.31+144136.03$ & $54242-2750-415$ & 17.85 & 3.102 & & $\mathrm{y}$ & $\mathrm{y}$ \\
\hline J150332.18+364118.06 & 52819-1352-194 & 17.85 & 3.263 & & $\mathrm{y}$ & $\mathrm{y}$ \\
\hline $\mathrm{J} 150923.38+243243.30$ & $53820-2155-383$ & 17.49 & 3.072 & $\mathrm{y}$ & $\mathrm{y}$ & $\mathrm{y}$ \\
\hline $\mathrm{J} 151352.52+085555.78$ & $53876-1719-173$ & 17.50 & 2.904 & & $\mathrm{y}$ & \\
\hline $\mathrm{J} 152553.89+513649.28$ & 52378-0795-245 & 16.84 & 2.882 & & & \\
\hline J164219.89+445124.01 & 52051-0629-463 & 17.88 & 2.882 & & $\mathrm{y}$ & $\mathrm{y}$ \\
\hline
\end{tabular}

Notes.

${ }^{a}$ The corresponding SDSS plate number, modified julian date, and fiber number for the object.

${ }^{b}$ SDSS redshift of the object.

${ }^{c}$ Absorption troughs detected at a similar velocity to $\mathrm{C}$ IV. 
We use the Si IV 1403 trough as a template for three reasons: (1) the larger separation of the Si IV doublet $\left(2000 \mathrm{~km} \mathrm{~s}^{-1}\right)$ compared to C IV $\left(500 \mathrm{~km} \mathrm{~s}^{-1}\right)$ produces well-separated Si IV troughs in most objects, which is not the case for the C IV doublet; (2) as we will show in Section 3, CIV is expected to be highly saturated when S IV is detected, while Si IV is less saturated; and (3) Si IV 1403 is the weaker of the Si IV doublet lines (by a factor of two) and therefore is the least saturated.

All three S IV objects show absorption troughs from Si IV, N v, $\mathrm{O}$ VI, Ly $\alpha$, and $\operatorname{Ly} \beta$. Two of the three objects show absorption from Al III and two from C II (SDSS J1145+3937 shows neither). SDSS J1509+2432 also shows other low-ionization species (e.g., Fe II $\lambda 1608$ and Si II $\lambda 1260$ ) and an excited Si II $\lambda 1265$ trough. One particular object in our sample, SDSS J1347+4654, has potentially saturated S IV absorption lines since it has similar residual intensity in both the resonance and excited state troughs. This can only occur if the lines are either saturated or if the electron number density of the outflowing gas is finely tuned to $10^{4.7} \mathrm{~cm}^{-3}$ (the value where the relative level populations of S IV and $\mathrm{SIV}^{*}$ are equal for $T=10^{4} \mathrm{~K}$ ), which makes the former scenario more likely. Relative level populations for the two S IV energy levels were computed from Equation (3.27) in Osterbrock \& Ferland (2006), which considers only collisional and radiative transitions. Atomic data for these computations were taken from version 2 of the XSTAR Atomic Database, described by Bautista \& Kallman (2001).

\subsection{Expanding the Search to Fainter Objects}

One concern with the above sample is basing conclusions on only three detections of S IV. In order to evaluate whether this result is representative, we also examine a fainter range in $r^{\prime}$ magnitude, from 18 to 18.5 . This magnitude range provides a sample of 348 objects with the proper redshift and velocity criteria. Of these 348 objects, we find eight firm detections and five weaker detections of S IV and S IV*. Thus, for this fainter sample, the detection percentage is between $2.3 \%$ and $3.7 \%$. This detection rate is consistent with the one we find in the brighter sample.

In addition, in the fainter sample of objects, we find one object (SDSSJ0746+3014) that shows evidence for a trough from the S IV $\lambda 1063$ without the presence of the $\mathrm{S} \mathrm{IV}^{*} \lambda 1073$ line (see Figure 2). This object shows no signs of troughs from lowionization species (e.g., $\mathrm{C}$ II and Si II). We note that the C IV in this object is significantly broader but is consistent with the velocity range of the S IV and Si IV absorption. Due to a nondetection of the excited state line, it is plausible that this outflow is situated at a distance several $\mathrm{kpc}$ from the central source.

\section{COMPARISON WITH DETECTION PERCENTAGE OF OTHER IONIC SPECIES}

\subsection{CIV}

Quasar absorption outflows are usually identified by the appearance of absorption from the C IV doublet $(\lambda \lambda 1548,1551)$ in their spectrum (Weymann et al. 1991; Crenshaw et al. 1999). The ease of $\mathrm{C}$ IV detection is due to the abundance of carbon, the strong oscillator strengths of these lines, the relatively low redshift in which the $\mathrm{C}$ IV doublet can be detected from groundbased observations, and the relatively high ionization parameter $\left(U_{\mathrm{H}}\right.$, see Section 5) common in UV outflows. The expected detection ratio between $\mathrm{C}$ IV and S IV is directly related to the optical depth ratio of their lines, which depends on the relative abundances $(\chi)$ of the elements, the ionic fraction of each

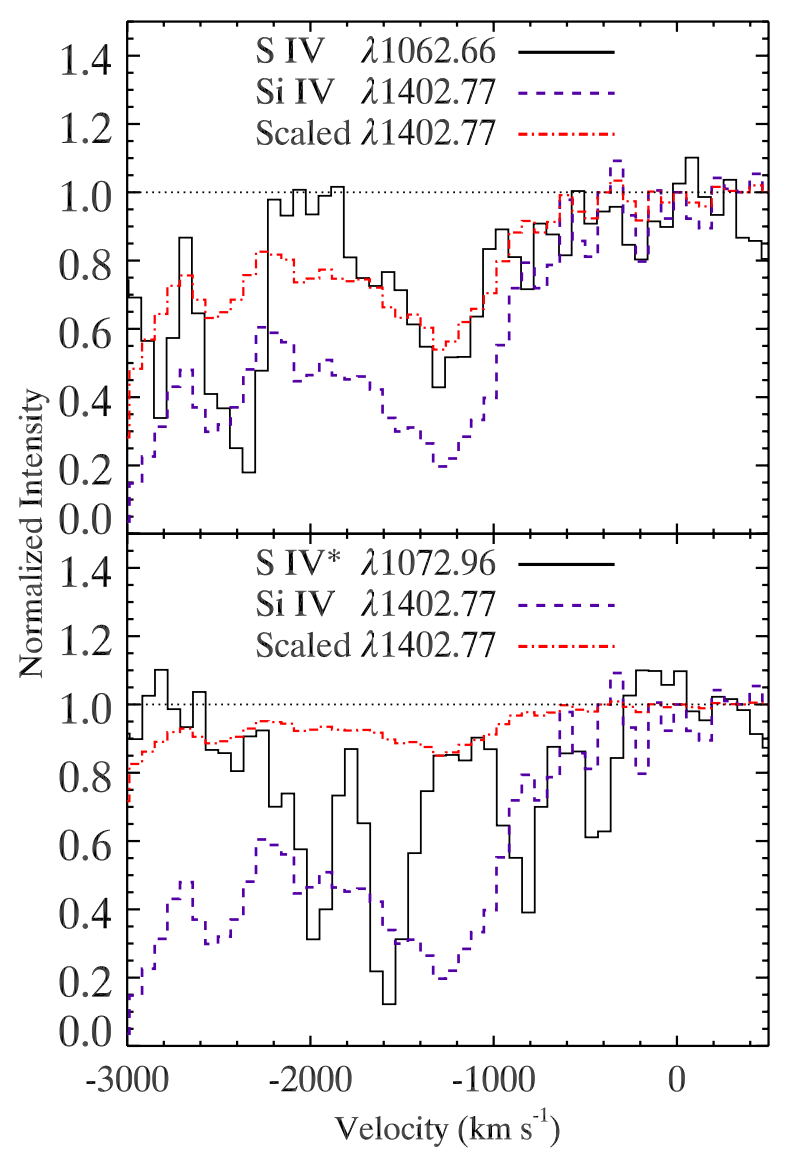

Figure 2. SDSSJ0746+3014. Top: trough profile match between Si IV and S IV. Bottom: same as top but for the $\mathrm{SIV}^{*}$ line, showing no, or very weak, $\mathrm{S} \mathrm{IV}^{*}$ trough using the Si IV template.

(A color version of this figure is available in the online journal.)

species as a function of $U_{\mathrm{H}}$ [hereafter: $F_{\text {ion }}\left(U_{\mathrm{H}}\right)$ ], and the ratio of the their oscillator strength $(f)$ times wavelength $(\lambda)$ :

$$
\begin{aligned}
\frac{\tau_{\mathrm{S}_{\text {IV }}}}{\tau_{\mathrm{C}_{\text {IV }}}}\left(U_{\mathrm{H}}\right) & =\frac{\chi(\mathrm{S})}{\chi(\mathrm{C})} \frac{F_{\mathrm{C}_{\text {IV }}}\left(U_{\mathrm{H}}\right)}{F_{\mathrm{S}_{\text {IV }}}\left(U_{\mathrm{H}}\right)} \frac{f_{\mathrm{S}_{\text {IV }}}}{f_{\mathrm{C}_{\text {IV }}}} \frac{\lambda_{\mathrm{S} \text { IV }}}{\lambda_{\mathrm{C}_{\text {IV }}}} \\
& =\frac{1}{16} \frac{F_{\mathrm{C}_{\text {IV }}}\left(U_{\mathrm{H}}\right)}{F_{\mathrm{S}_{\text {IV }}}\left(U_{\mathrm{H}}\right)} \frac{0.05}{0.19} \frac{1063}{1548} \\
& =0.011 \frac{F_{\mathrm{C}_{\text {IV }}}\left(U_{\mathrm{H}}\right)}{F_{\mathrm{S}_{\text {IV }}}\left(U_{\mathrm{H}}\right)} .
\end{aligned}
$$

This is a limit on the detection ratio due to issues connected with the LOS covering of the background source (see Section 3.2).

Figure 3 shows the relative optical depth for $\mathrm{C}$ IV and S IV (as well as for Si IV and C II) as a function of $U_{\mathrm{H}}$. The shapes of the optical depth curves as a function of $U_{\mathrm{H}}$ are singularly dependent on $F_{\text {ion }}\left(U_{\mathrm{H}}\right)$ (all the other variables in Equation (2) simply scale the ratio by a single factor). We observe that the relative ionization fractions for S IV and C IV are roughly constant, peaking near $\log U_{\mathrm{H}}=-2.1$. This behavior is relatively insensitive to changes in the spectral energy distribution (SED). To demonstrate this, we also calculate the ionization fractions with a "UV-Soft" SED (see Dunn et al. 2010) and find only small changes (less than $0.1 \mathrm{dex}$ ) for this significantly softer SED (see online Figure 4). Therefore, the absorption troughs from S IV and CIV are likely to arise from the same physical region of any given outflow. We note that the models shown in Figure 3 are optically thin to all bound-free transitions, where we used a 
Table 2

Measurements of S IV Troughs

\begin{tabular}{|c|c|c|c|c|c|c|c|c|c|c|}
\hline \multirow[t]{2}{*}{ Object } & \multicolumn{3}{|c|}{$\mathrm{C}_{\text {IV }} \lambda \lambda 1548,1550$} & \multicolumn{4}{|c|}{ S IV $\lambda 1063$} & \multicolumn{3}{|c|}{$\mathrm{S}_{\mathrm{IV}} * \lambda 1073$} \\
\hline & $\begin{array}{c}\text { FWHM } \\
\left(\mathrm{km} \mathrm{s}^{-1}\right)\end{array}$ & $I_{r}{ }^{\mathrm{a}}$ & $\sigma_{I_{r}}{ }^{\mathrm{a}}$ & $\begin{array}{c}\text { FWHM } \\
\left(\mathrm{km} \mathrm{s}^{-1}\right)\end{array}$ & $\begin{array}{l}\text { Velocity } \\
\left(\mathrm{km} \mathrm{s}^{-1}\right)\end{array}$ & $I_{r}{ }^{\mathrm{a}}$ & $\sigma_{I_{r}}{ }^{\mathrm{a}}$ & $\begin{array}{c}\text { FWHM } \\
\left(\mathrm{km} \mathrm{s}^{-1}\right)\end{array}$ & $I_{r}{ }^{\mathrm{a}}$ & $\sigma_{I_{r}}{ }^{\mathrm{a}}$ \\
\hline SDSSJ1145+3937 & $4720 \pm 30$ & $<0.01$ & 0.03 & $1340_{-30}^{+130}$ & -2490 & 0.33 & 0.05 & $1510_{-110}^{+260}$ & 0.42 & 0.05 \\
\hline SDSSJ1347+4654 & $3770 \pm 30$ & 0.02 & 0.04 & $1660_{-50}^{+260}$ & -1480 & 0.18 & 0.09 & $1610_{-140}^{+160}$ & 0.17 & 0.08 \\
\hline SDSSJ1509+2432 & $5240 \pm 20$ & 0.05 & 0.02 & $2160_{-130}^{+260}$ & -1110 & 0.36 & 0.09 & $2590_{-120}^{+80}$ & 0.23 & 0.08 \\
\hline
\end{tabular}

Note. ${ }^{a}$ Normalized intensity.

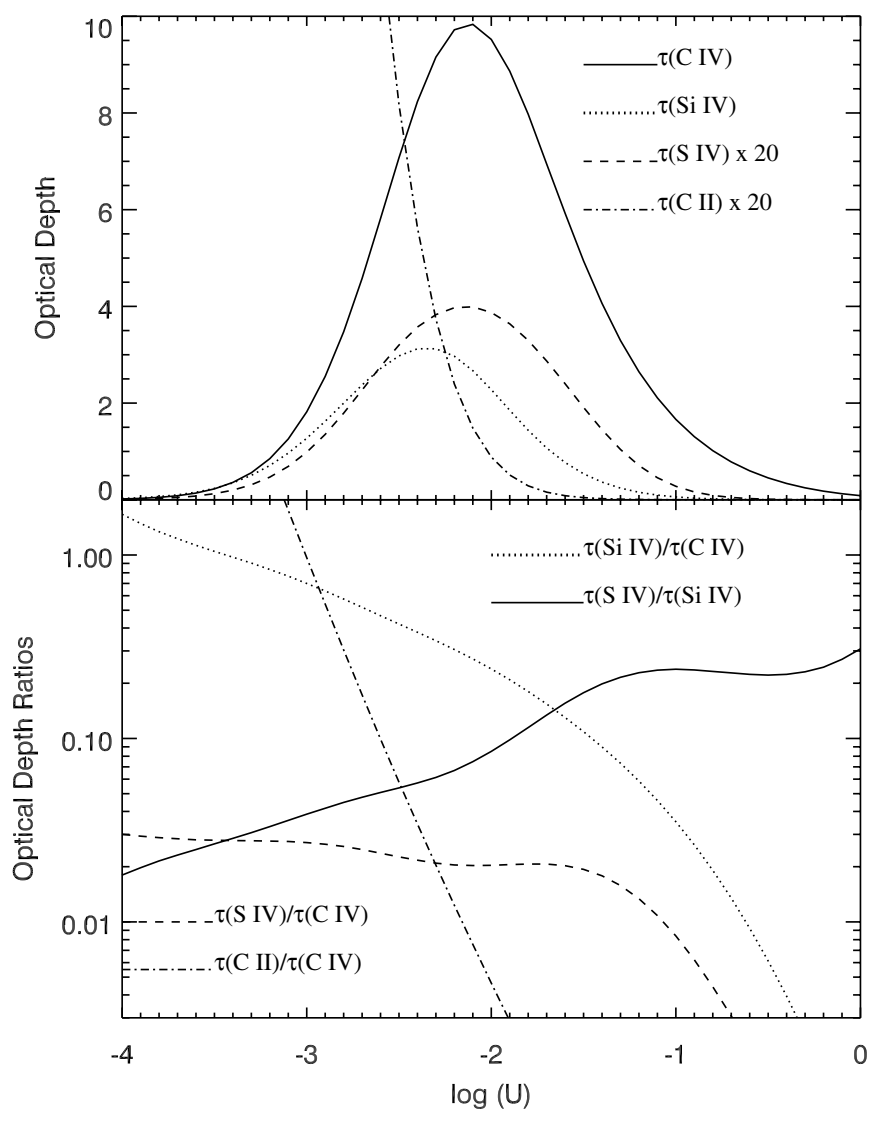

Figure 3. Expected optical depth for CIV, Si IV, S IV, and C II lines, from Equation (2), assuming solar abundances (Lodders et al. 2003) and normalizing to $\tau_{\max }(\mathrm{C}$ IV $)=10$. We used an oscillator strength of 0.05 for S IV $\lambda 1063$ (Hibbert et al. 2002), 0.19 for C IV $\lambda 1548,0.52$ for S IV $\lambda 1393$, and 0.13 for C II $\lambda 1334$ (Kurucz \& Bell 1995). Ionization fractions were calculated with the photoionization code CLOUDY using a Mathews \& Ferland (1987) SED.

small total column density $\left(N_{\mathrm{H}}=10^{17} \mathrm{~cm}^{-2}\right)$ to guarantee this result in all possible cases.

Our calculated ratio of expected optical depth depends linearly on the $\mathrm{S} / \mathrm{C}$ abundance ratio, which here we adopt as solar $(\approx 1 / 16$; Lodders et al. 2003). Carbon is mostly produced in lowand intermediate-mass asymptotic giant branch stars, while sulfur is an $\alpha$ element and is produced in supernova explosions from massive stars $\left(M>8 M_{\odot}\right)$. Observations of Galactic halo stars confirm that the sulfur abundance follows that of silicon and magnesium (e.g., Nissen et al. 2007 and references therein). Thus, models of galactic chemical evolution vs. metallicity (e.g., Kobayashi et al. 2006) predict variations of less than a factor of three for $\mathrm{S} / \mathrm{C}$, with respect to the solar abundance ratio.

The shallowest $\mathrm{S}$ IV trough in our sample has a residual intensity of $I_{r}=0.36$ (see Figure 1 and Table 2), and therefore an

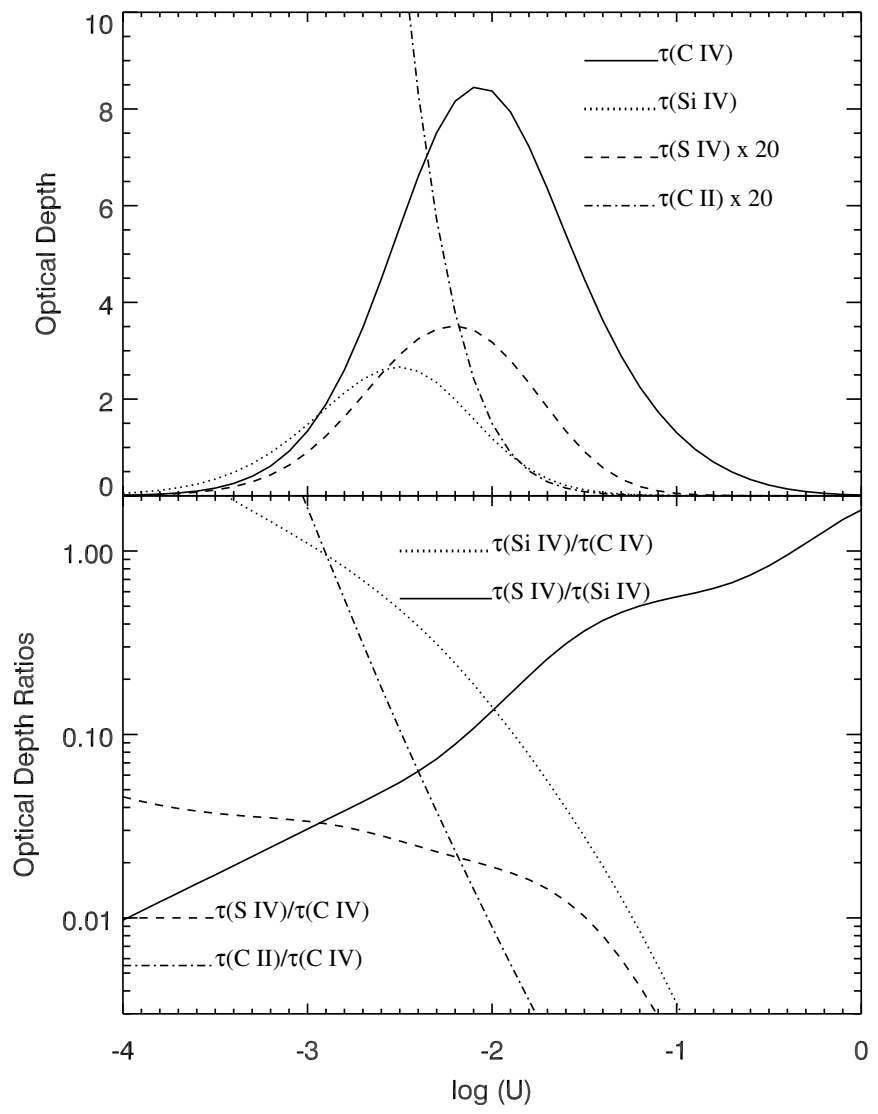

Figure 4. Same as Figure 3, but using a "UV-Soft" SED (Dunn et al. 2010). This figure demonstrates that the ionization fraction ratio of C IV to S IV is relatively insensitive to changes in the SED.

apparent optical depth of $\tau_{\text {app }} \approx 1$. If we assume that we detect all $\mathrm{S}$ IV troughs in quasars with $\tau_{\text {app }}(\mathrm{S}$ IV) $>1.0$ (deep enough to be detected in the Ly $\alpha$ forest) and that the covering fraction ratio of the two ions is similar (see Section 3.2), then from Figure 3 it is evident that $\tau(\mathrm{C}$ IV $) \gtrsim 50$, which is their optical depth ratio. In Section 3.1, we showed that in our sample the frequency of C IV detection is $14 \%$ and that of S IV is $1.9 \%$, which yields a ratio of $\mathrm{S}$ IV to C IV detection of $1 / 7$ or approximately $14 \%$. The large disparity in the optical depth ratio explains why we do not detect $\mathrm{S}$ IV absorption troughs in every object with a C IV trough and simply implies that $\tau(\mathrm{C}$ IV $) \lesssim 50$ in $\sim 86 \%$ of the $\mathrm{C}_{\text {IV }}$ objects.

\subsection{Absorption Material Distribution}

One additional factor that we cannot easily quantify that would affect the number of expected detections is the average LOS covering. In cases where there is LOS partial covering of the background source, the troughs of S IV will be shallower 
for the same optical depth. Thus, we would expect to detect fewer S IV absorption systems compared to $\mathrm{C}$ IV systems when the LOS covering factor $\left(C_{f}\right)$ is small. Equation (2) describes the ratio of optical depth between S IV and CIV, assuming complete covering. This requires a correction due to the ratio of the average LOS covering $\left(\left\langle C_{f}\right\rangle\right)$ between S IV and C IV if they are different, which can be the case between ions (Arav et al. 1996; Hamann et al. 2001), even those that form under similar conditions. There are two approaches to determine $\left\langle C_{f}\right\rangle$ for a sample of objects.

The first method to determine $\left\langle C_{f}\right\rangle$ is to examine separated doublets (Hamann et al. 1997) and calculate the covering factors for each object. However, the C IV doublets are blended due to our selection criteria and the S IV line is not a member of a doublet, which precludes this method for both ionic species. The second approach is to measure the average residual intensity for known saturated lines. In other words, the LOS covering is defined by $\left\langle C_{f}\right\rangle=1-\left\langle I_{\min }\right\rangle$ (Crenshaw et al. 2003), where $I_{\min }$ is the minimum normalized intensity in a saturated trough. The depths of the C IV absorption troughs for our sample yield $\left\langle C_{f}\right\rangle \approx 0.80$. We cannot guarantee an accurate determination of $\left\langle C_{f}\right\rangle$ for the $\mathrm{S}$ IV lines because only one object shows troughs with signs of saturation; $C_{f} \approx 0.82$ for that case. If we assume that the other two objects are also saturated, then $\left\langle C_{f}\right\rangle$ is 0.71 for S IV. If the troughs in these two objects are not saturated, then this becomes a lower limit. In either case, the difference between the $\left\langle C_{f}\right\rangle$ for S IV and C IV is not large $(\sim 10 \%-20 \%)$.

The caveat with the value of $\left\langle C_{f}\right\rangle=0.71$ for $S_{\text {IV }}$ is that this value can be significantly smaller. For the case where $\left\langle C_{f}\right\rangle$ is different for the two ions, the main qualitative difference is that there are more S IV systems our survey will not detect. Therefore, our detection fraction becomes a stringent lower limit on the number of systems with S IV (we discuss the implications of this in Section 4.1). This is because $C_{f}$ for C IV is always larger than that of S IV (as evident both in this sample and in general as S IV is a much rarer species compared to C IV, e.g., Arav et al. 1999, p. 0946). For any case where $C_{f}$ of $\mathrm{S}$ IV is smaller than $\sim 0.5$, we lose the ability to discern $\mathrm{S}$ IV troughs from the Ly $\alpha$ forest. Thus, when $\left\langle C_{f}\right\rangle$ is significantly smaller than 1.0, we will miss more such outflows in our SDSS survey than in cases where $C_{f}(\mathrm{C}$ IV $) \simeq C_{f}(\mathrm{~S}$ IV $) \sim 1$ and our detection fraction is a lower limit. Such systems may reveal themselves in data with higher spectral resolution and $\mathrm{S} / \mathrm{N}$.

\subsection{Comparison to Outflows with Si IV and C II, and Synthesis}

In Section 3.1, we found that $11 \%$ of the objects in our sample have detectable Si IV troughs. Comparing the detection rate of S IV with Si IV, the fraction of objects with Si IV for which we detect $\mathrm{S}$ IV is approximately $17 \%$. Figure 3 shows that $F_{\text {Si IV }}\left(U_{\mathrm{H}}\right)$ peaks at lower $U_{\mathrm{H}}$ values than that of $\mathrm{C}$ IV and $\mathrm{S}$ IV as a function of $U_{\mathrm{H}}$. Therefore, the predicted ratio of $\tau_{\mathrm{Si} \text { IV }} / \tau_{\mathrm{S} \text { IV }}$ changes from 25 to 5 as $\log \left(U_{\mathrm{H}}\right)$ varies from -3 to -1.3 , and has a value of 13 for $\log \left(U_{\mathrm{H}}\right)=-2.1$, where $F_{\mathrm{S} \mathrm{IV}}\left(U_{\mathrm{H}}\right)$ is at the maximum. Similarly, the predicted $\tau_{\mathrm{Si} \text { IV }} / \tau_{\mathrm{C} \text { IV }}$ changes from 0.7 to 0.07 as $\log \left(U_{\mathrm{H}}\right)$ varies from -3 to -1.3 (we limit $-3<\log \left(U_{\mathrm{H}}\right)<-1.3$, since beyond this range $\tau_{\mathrm{S}}$ IV falls below $25 \%$ of its maximum value).

Both expected behaviors are consistent with the observed detection rates in our sample. The predicted $\tau_{\mathrm{Si} \text { IV }}$ is smaller than that of Civ, therefore fewer objects should show Si IV troughs compared to $\mathrm{C}$ IV ones. Whereas, the predicted $\tau_{\mathrm{Si} \text { IV }}$ is larger than that of S IV, therefore more objects should show $\mathrm{Si}$ IV troughs compared to $\mathrm{S}$ IV ones. This explanation also holds semi-quantitatively; for the $U_{\mathrm{H}}$ range of interest, $\tau_{\mathrm{Si} \text { IV }} / \tau_{\mathrm{S} \text { IV }}$ is significantly larger than $\tau_{\mathrm{C} \text { IV }} / \tau_{\mathrm{Si} \text { IV }}$ (by a factor ranging from 18 to 1 , for $\left.-3<\log \left(U_{\mathrm{H}}\right)<-1.3\right)$. For this $U_{\mathrm{H}}$ range, we therefore expect the fraction of Si IV detections to be in between, but closer to that of $\mathrm{C}$ IV than to $\mathrm{S}$ IV; in good agreement with our results of sample detection percentages: $14 \%$ for C IV, $11 \%$ for Si IV, and $1.9 \%$ for S IV.

$\mathrm{C}$ II outflow troughs are detected in 13 of our sample objects $(8.3 \%)$. Figure 3 shows that $F_{\mathrm{C} \text { II }}\left(U_{\mathrm{H}}\right)$ peaks at much lower values that that of $\mathrm{Si} I V$ and $\mathrm{C}$ IV and drops quickly over the $-3<\log \left(U_{\mathrm{H}}\right)<-1.3$. Combining information for the detection of all four ions with the expected optical depth of their troughs and assuming that the plasma is optically thin to boundfree transitions (see Figure 3), a simple toy model can explain the difference in detection percentage.

1. All four ionic troughs come from the same outflows.

2. All outflows have $\log \left(U_{\mathrm{H}}\right) \sim-2.4$.

3. There is a wide distribution of $\tau_{\mathrm{C} \text { IV }}$ with $\sim 14 \%$ of the outflows having $\tau_{\mathrm{C}_{\text {IV }}}>50$.

Assumption (2) is probably the weakest, as a range of $U_{\mathrm{H}}$ values is expected. Nonetheless, the implication of this toy model is that with the same $\Omega$ for all four ions, we can explain the differing detection percentage using only the expected optical depth of the lines.

\section{DISCUSSION}

\subsection{Global Covering for Quasar Absorption Outflows}

As noted in Section 1, the mass flux and kinetic luminosity of an outflow are linearly dependent on the solid angle subtended by the wind as viewed from the quasar $(\equiv 4 \pi \Omega)$. Because there is no direct spectroscopic determination for $\Omega$, the common method is to statistically determine an average global covering $\left(C_{G}\right)$ via the detection fraction of absorbers in spectroscopic surveys and use it as an approximation of $\Omega$ (see Section 3.2 for discussion of partial LOS covering). For example, as mentioned in the Section 1, Hewett \& Foltz (2003) and Dai et al. (2008) find that C IV BALs appear in approximately $20 \%$ of all quasars. This can be interpreted as either $\Omega \approx C_{G} \sim 0.2$ in every quasar, or that $\sim 20 \%$ of all quasars have their sky completely covered $(\Omega=1)$, or that all quasars have $C_{G} \sim 1$ for $20 \%$ of their duty cycle. For the outflows' integrated influence over time for an ensemble of quasars, all three interpretations yield similar amounts of injected mass and energy.

Traditionally, the global covering factor for a specific type of outflow (e.g., outflows with troughs from C IV) is determined statistically via the product of the average LOS covering factor $\left(\left\langle C_{f}\right\rangle\right)$ and the fraction of absorbers detected $(f$; Crenshaw et al. 2003). The values for $\left\langle C_{f}\right\rangle$ between S IV and C IV are plausibly similar as we show in Section 3.2 (given the assumptions outlined). Furthermore, if we are missing detections of S IV due to low $C_{f}$ in objects (as explained in Section 3.2), then the actual detection fraction $f$ is larger. Thus, regardless of the uncertainty in $\left\langle C_{f}\right\rangle$, the global covering for S IV does not drastically affect the $C_{G}$ (see Section 4.2).

Much research has been devoted to finding $f$, and thus $C_{G}$, for various ions in outflows. Crenshaw et al. (1999) examined C IV absorption in a sample of low- $z$ AGNs observed with the Hubble Space Telescope and found that outflows in the UV in these objects were common, appearing in approximately $50 \%$ of the objects in their sample. Dunn et al. (2007) found a similar detection rate for low- $z$ AGNs using absorption lines from $\mathrm{O}$ VI 
observed with the Far-Ultraviolet Spectroscopic Explorer. In higher redshift objects, Ganguly et al. (2007), searching through spectra from the SDSS, confirmed the Hewett \& Foltz (2003) result.

\subsection{Global Covering for S IV Outflows}

In Section 2.2, we showed that the conservative detection rate of S IV outflows is $1.9 \%$. Because we are observing SIV troughs in the optical, we need to apply a $k$-correction, which compensates for differences in the SED in the far UV. The factor for the $k$-correction is $22 / 15$ as found by Hewett \& Folz (2003), which also applies to our current survey because the SDSS spectra cover the same rest wavelength range for these objects. Using the new value, we can directly compare the optical detection rate to the actual fraction of quasars with $\mathrm{C}$ IV BALs. The resulting $2.8 \%$ can be used as a stringent lower limit on $C_{G}$ of S IV outflows. The upper limit is again that the global covering is equal to $\mathrm{C}$ IV, with $C_{G} \approx 0.20$. As we showed in Section 3, the detection percentage of C IV, Si IV, C II, and S IV can all be explained straightforwardly by the expected optical depth for the lines of each species assuming that they arise from the same outflow and have similar $C_{f}$, which is plausible given the small range for the ratio between S IV and C IV. Therefore, it is probable that $\mathrm{C}$ IV and S IV troughs arise from the same outflows and thus have the same $C_{G}$ and $\Omega$.

It is also plausible that the $N_{\mathrm{H}}$ for both outflows' manifestations is similar and the cases where we do not detect S IV troughs simply have a higher ionization parameter. Since the fraction of both C IV and S IV drops for $\log \left(U_{\mathrm{H}}\right)>-2.1$, the large difference in optical depth can explain why the S IV troughs disappear while we would still detect C IV (see Figure 3). Indeed, many quasar outflows are known to have a higher $U_{\mathrm{H}}$ value, e.g., $\log \left(U_{\mathrm{H}}\right) \sim-0.8$ for the outflow seen in quasar J212329.46-005052.9 (Hamann et al. 2010) and $\log \left(U_{\mathrm{H}} \sim-1.4\right.$ in the quasar FBQS J1151+3822 (Leighly et al. 2011). If, however, $\log \left(U_{\mathrm{H}}\right)$ for all $\mathrm{C}$ IV outflows is in a narrow range around -2.1 , then outflows that show $\mathrm{S}$ IV troughs will have a larger $N_{\mathrm{H}}$, which will be needed to explain the S IV detection. In such a scenario, the $S$ IV outflows will tend to have higher $\dot{M}$ and $\dot{E}_{k}$ than the majority of C IV outflows (see Equation (1)).

\section{SUMMARY AND CONCLUSIONS}

We establish the detection percentage of S IV outflows in quasar spectra for troughs arising from both resonance $(\lambda 1063)$ and the excited state transition ( $\lambda 1073$ ) lines. In our bright (brighter than $r^{\prime}$ magnitude of 18) SDSS sample of 156 quasars with $z>2.8$ (the redshift requirement to detect $S_{\text {IV }} \lambda 1063$ in the SDSS spectra), 22 have C IV outflows and 3 of these show $\mathrm{S}$ IV troughs. This yields detection rates of $21 \%$ for $\mathrm{C}$ IV and $2.8 \%$ for $\mathrm{S}$ IV, after applying a $k$-correction. We also examine a similar sample of 348 quasars with an $r^{\prime}$ magnitude between 18 and 18.5 and find that, given the weaker $\mathrm{S} / \mathrm{N}$, the percentage of $\mathrm{S}$ IV detection is consistent with that of the bright sample.

A detailed comparison of the expected optical depth $(\tau)$ between $C_{\text {IV }} \lambda 1548$, Si IV $\lambda 1393, C_{\text {II }} \lambda 1334$, and S IV $\lambda 1063$ demonstrates that the detected percentage of each ion in our sample is in good agreement with the ratio of expected $\tau$ of the troughs. An analysis of $\left\langle C_{f}\right\rangle$ for the objects with S IV troughs shows that the difference in detection fraction is plausibly small and that a disparate $\left\langle C_{f}\right\rangle$ between the two ions does not strongly affect the determination of global covering. We therefore conclude that all of these lines arise from the same outflows and that the fraction of solid angle subtended by them $(\Omega)$ is the same for C IV and S IV outflows, where the difference in detection percentage can be fully explained by the large expected $\tau_{\mathrm{C}_{\text {IV }}} / \tau_{\mathrm{S} \text { IV }} \sim 50$.

We intend to follow up all three of the objects in our bright sample with high resolution and high-S/N spectra in an effort to determine radial distances of the outflows. However, in Section 3, we find one object with a trough likely to be S IV whose profile is similar to the Si IV trough, yet there is no evidence of a $\mathrm{SIV}^{*}$ trough. Plausibly, this outflow could be situated at a distance of several kpc, much like the distances we find for the Fe II and Si II outflows (see Table 3 in Arav et al. 2012 , in preparation). Should this distance be confirmed with a higher quality spectrum, it would lend support to the assertion that the outflows where we detect Fe II and Si II troughs are the same outflows as those without but viewed through a high opacity LOS that allows for the formation of singly ionized species (see Hall et al. 2003; Dunn et al. 2010; Arav et al. 2012, in preparation).

We acknowledge support from NSF grant AST 0837880 and from NASA LTSA grant NAG5-12867. We also thank Kirk Korista, Mike Crenshaw, and Pat Hall for their insightful suggestions and discussions.

\section{APPENDIX}

\section{IV AND S IV TROUGH MEASUREMENTS}

We measure the following trough properties from the SDSS spectra for the SIV outflows: residual intensity (using our estimate of the continuum flux level), central velocity, and the FWHM of the absorption trough, and present these in Table 2. Measurements of the S IV and S IV* troughs are complicated by contamination from $\operatorname{Ly} \alpha$ forest lines. We therefore outline our methods for determining these properties.

The first parameter we determine from the data is the central velocity of the outflow. We recognize that Ly $\alpha$ intervening absorbers can potentially affect the centroid of the S IV and $\mathrm{S} \mathrm{VI}$ * troughs, which makes these troughs less than ideal for determining the central velocity of the absorption. We also cannot use the $\mathrm{C}$ IV troughs due to our velocity width restrictions (C IV with FWHM larger than $500 \mathrm{~km} \mathrm{~s}^{-1}$ ), which leads to the individual troughs self-blending and rendering determinations of the central velocity impossible. Thus, we use templates of the kinematically coincident Si IV trough lines (shown in Figure 1) to determine the velocity. In two of the three objects, the troughs are separated enough in velocity to allow such a determination. The Si IV troughs in SDSSJ1509+2432 suffer from self-blending as the FWHM of the troughs is over $2000 \mathrm{~km} \mathrm{~s}^{-1}$. Therefore, we resort to using the average velocity centroid of S IV and S IV* in that particular object. We do note that because we use the average of the two troughs, the effective random shifts from Ly $\alpha$ forest lines should statistically be reduced.

The second parameter we measure is the residual intensity $\left(I_{R}\right)$, which is defined as the normalized flux level at the deepest part of the trough. We employ two methods for determining the $I_{R}$ of the SIV troughs. First, for the broader S IV and $\mathrm{S}$ IV* troughs, we approximate the residual intensity $\left(I_{R}\right)$ as the average depth across the bottom of the trough. This is necessary because the sharp variations in the trough are likely due to Ly $\alpha$ forest contamination that creates a lower $I_{R}$ than the real S IV troughs. We estimate the error in this measurement by adding in quadrature the standard deviation of the points in the bottom 
of the trough and the respective average intensity error for those points. For the narrower S IV troughs (i.e., SDSSJ1145+3937), as well as their respective C IV troughs, we can only take the $I_{R}$ as the deepest point in the trough. To determine the error in the $I_{R}$ measurement we simply use the SDSS provided uncertainty. Finally, we use $I_{R}$ to calculate the flux at half-maximum and measure the width of the trough. We use the uncertainty in $I_{R}$ to determine the range of acceptable half-maximum within the trough. This range translates to an upper and lower limit for the FWHM of the trough determined by the walls of the trough at the extrema.

\section{REFERENCES}

Arav, N. 1996, ApJ, 465, 617

Arav, N., de Kool, M., Korista, K. T., et al. 2001, ApJ, 561, 118

Arav, N., Korista, K. T., de Kool, M., Junkkarinen, V. T., \& Begelman, M. C. 1999, ApJ, 516, 27

Arav, N., Moe, M., Costantini, E., et al. 2008, ApJ, 681, 954

Bautista, M. A., Dunn, J. P., Arav, N., et al. 2010, ApJ, 713, 25

Bautista, M. A., \& Kallman, T. R. 2001, ApJS, 134, 139

Bechtold, J. 1994, ApJS, 91, 1

Crenshaw, D. M., Kraemer, S. B., Boggess, A., et al. 1999, ApJ, 516, 750

Crenshaw, D. M., Kraemer, S. B., \& George, I. M. 2003, ARA\&A, 41, 117

Dai, X., Shankar, F., \& Sivakoff, G. R. 2008, ApJ, 672, 108

Dai, X., Shankar, F., \& Sivakoff, G. R. 2010, ApJ, submitted

Danforth, C. W., \& Shull, J. M. 2008, ApJ, 679, 194

Dunn, J. P., Bautista, M., Arav, N., et al. 2010, ApJ, 709, 611
Dunn, J. P., Crenshaw, D. M., Kraemer, S. B., \& Gabel, J. R. 2007, AJ, 134, 1061

Ganguly, R., Brotherton, M. S., Cales, S., et al. 2007, ApJ, 665, 990

Hall, P. B., Hutsemékers, D., Anderson, S. F., et al. 2003, ApJ, 593, 189

Hamann, F., Barlow, T. A., Chaffee, F. C., Foltz, C. B., \& Weymann, R. J. 2001, ApJ, 550, 142

Hamann, F., Barlow, T. A., Junkkarinen, V., \& Burbidge, E. M. 1997, ApJ, 478, 80

Hamann, F., Kanekar, N., \& Prochaska, J. X. 2010, MNRAS, 410, 1957

Hewett, P. C., \& Foltz, C. B. 2003, AJ, 125, 1784

Hibbert, A., Brage, T., \& Fleming, J. 2002, MNRAS, 333, 885

Kobayashi, C., Umeda, H., Nomoto, K., Tominaga, N., \& Ohkubo, T. 2006, ApJ, 653,1145

Korista, K. T., Bautista, M. A., Arav, N., et al. 2008, ApJ, 688, 108

Kraemer, S. B., Crenshaw, D. M., Gabel, J. R., et al. 2006, ApJS, 167, 161

Kurucz, R. L., \& Bell, B. 1995, Atomic Line List (Kurucz CD-ROM; Cambridge, MA: Smithsonian Astrophysical Observatory)

Leighly, K. M., Dietrich, M., \& Barber, S. 2011, ApJ, 728, 94

Leighly, K. M., Hamann, F., Casebeer, D. A., \& Grupe, D. 2009, ApJ, 701, 176

Lodders, K. 2003, ApJ, 591, 1220

Mathews, W. G., \& Ferland, G. J. 1987, ApJ, 323, 456

Moe, M., Arav, N., Bautista, M. A., \& Korista, K. T. 2009, ApJ, 706, 525

Nissen, P. E., Akerman, C., Asplund, M., et al. 2007, A\&A, 469, 319

Osterbrock, D. E., \& Ferland, G. J. (ed.) 2006, Astrophysics of Gaseous Nebulae and Active Galactic Nuclei (Mill Valley, CA: University Science Books)

Outram, P. J., Chaffee, F. H., \& Carswell, R. F. 1999, MNRAS, 310, 289

Schneider, D. P., Richards, G. T., Hall, P. B., et al. 2010, AJ, 139, 2360

Trump, J. R., Hall, P. B., Reichard, T. A., et al. 2006, ApJS, 165, 1

Weymann, R. J., Morris, S. L., Foltz, C. B., \& Hewett, P. C. 1991, ApJ, 373, 23

York, D. G., Adelman, J., Anderson, J. E., Jr., et al. 2000, AJ, 120, 1579 